\title{
A CHAOTIC FUNCTION WITH A SCRAMBLED SET OF POSITIVE LEBESGUE MEASURE
}

\author{
J. SMÍTAL ${ }^{1}$
}

\begin{abstract}
There is a continuous map of the unit interval $I$ which is chaotic in the sense of $\mathrm{Li}$ and Yorke and which has a scrambled set of Lebesgue measure arbitrarily close to 1 .
\end{abstract}

If $f$ is a continuous self-mapping of $I=[0,1]$ then by $f^{n}$ we always denote the $n$th iterate of $f$. We say that $\left\{f^{n}(x)\right\}_{n=1}^{\infty}$, for any $x \in I$, is a sequence generated by $x$; it is said to be asymptotically periodic provided its cluster set is finite. Further, $f$ is chaotic if there is an uncountable set $S \subset I$ (a scrambled set) of points which generate sequences not asymptotically periodic and such that, for every $x, y \in S$, $x \neq y$,

$$
\limsup _{n \rightarrow \infty}\left|f^{n}(x)-f^{n}(y)\right|>0
$$

and

$$
\liminf _{n \rightarrow \infty}\left|f^{n}(x)-f^{n}(y)\right|=0
$$

(cf [2]; for more details see e.g. [5]).

In the literature there are known some examples of functions which have only scrambled sets of zero Lebesgue measure (cf. [3 or 5], among others), but there is no known example of such a set having a positive Legesgue measure. In [4] it was shown that the hat function $f(x)=1-2\left|x-\frac{1}{2}\right|$ on $I$ has a nonmeasurable scrambled set, but no set of positive measure. In the present note we prove the following

THEOREM. For every $\varepsilon>0$ there is a chaotic continuous self-mapping $f$ of the unit interval $I=[0,1]$ which has a scrambled set $S$ of Lebesgue measure greater than $1-\varepsilon$.

We prove the theorem in several steps, beginning with the following preliminary construction.

Let $\left\{a_{n}\right\}_{n=0}^{\infty}$ be an arbitrary decreasing sequence of positive numbers with $a_{0}=$ 1. For every $k$. let $I_{k}$ denote the open interval $\left(a_{k+1}, a_{k}\right)$. In every interval $I_{k}$ choose an infinite sequence of disjoint closed intervals $\left\{I_{k j}\right\}_{j=0}^{\infty}$ with the property $x<y$ for every $x \in I_{k j}$ and $y \in I_{k, j+1}$. Let $g$ be a continuous mapping $I \rightarrow I$ such that $g$ is linear on every interval $I_{j k}$ and such that $g\left(I_{0 k}\right)=I_{k+1,0}$ and $g\left(I_{j k}\right)=I_{j-1, k+1}$ for $j \neq 0$. In other words, $g$ maps the intervals $I_{j k}$ as follows:

$$
I_{00} \rightarrow I_{10} \rightarrow I_{01} \rightarrow I_{20} \rightarrow I_{11} \rightarrow I_{02} \rightarrow I_{30} \rightarrow I_{21} \rightarrow I_{12} \rightarrow I_{03} \rightarrow \cdots .
$$

Received by the editors August 10, 1983.

1980 Mathematics Subject Classification. Primary 26A18; Secondary 54H20.

${ }^{1}$ Supported in part by National Sciences and Engineering Research Council of Canada Grant A-2972.

(C) 1984 American Mathematical Society $0002-9939 / 84 \$ 1.00+\$ .25$ per page 
The above quoted construction of $g$ is a slight modification of an example due to B. Barna [1].

Since the proof of our theorem is rather complicated, we give first an outline of the main idea. By the following Lemmas 1 and 3 , for arbitrary numbers $x$ and $y$ belonging to two different intervals $I_{i j}, I_{k s}$ the conditions (1) and (2) are satisfied with $f$ replaced by $g$. However, for $x, y$ from the same interval $I_{i j}$, unfortunately, $\lim _{n \rightarrow \infty}\left|g^{n}(x)-g^{n}(y)\right|=0$. Thus the desired function $f$ should be a modification of $g$ with the following properties: The scrambled set $S$ is a nowhere dense perfect subset of $I_{00}$. For every interval $G \subset I_{00}$ contiguous to $S$ something like shift operation is used: If $A$ and $B$ are the left and right parts of $S$ relative to $G$, then there is an index $u$ such that $f^{u}$ maps $A$ and $B$ into two different intervals $I_{i j}$ 's. More exactly, for some $k=k(G), f^{u}(A)=g^{u+k}(A)$ and $f^{u}(B)=g^{u+k+1}(B)$. Now using the properties of $g$ we can find numbers $m, n$ with $u<m<n$ such that

$$
\operatorname{dist}\left[f^{m}(A), f^{m}(B)\right]=\operatorname{dist}\left[g^{m+k}(A), g^{m+k+1}(B)\right] \geq \operatorname{dist}\left[g^{m+k}\left(I_{00}\right), g^{m+k}\left(I_{10}\right)\right]
$$

is large and $\operatorname{diam}\left[f^{n}(A) \cup f^{n}(B)\right]$ is small. Then $B$ is shifted "back", i.e. there is some $v>n$ such that $f^{v}(A)=g^{v+k+1}(A)$ and $f^{v}(B)=g^{v+k+1}(B)$. Moreover, for a given $G$ there are infinitely many of such indices $u, v$. This implies that for $x, y \in S$, which are separated by $G,(1)$ and (2) are true.

Now we can proceed with the proof. The following property of Barna's function $g$ plays an important role in the sequel.

LEMMA 1. If $I_{i j} \neq I_{k s}$ then there is an abitrarily large index $n$ with

$$
\operatorname{dist}\left[g^{n}\left(I_{i j}\right), g^{n}\left(I_{k s}\right)\right]>\eta
$$

where $\eta$ is the length of the interval $I_{00}$.

Proof. Assume e.g. that $g^{m}\left(I_{i j}\right)=I_{k s}$, for some $m>0$, and let $M$ be an arbitrary number. Choose $n>M$ such that $g^{n}\left(I_{i j}\right)=I_{0 p}$ and $g^{n+m}\left(I_{i j}\right)=$ $g^{n}\left(I_{k s}\right)=I_{q r}$, where $p, q, r$ are suitable numbers with $q \neq 0$. It is easy to see that such an $n$ does exist. But then $I_{00}$ lies between $I_{q r}$ and $I_{0 p}$ and the inequality follows.

For simplicity, we shall use the following notation:

$$
J_{0}=I_{00} \quad \text { and } \quad J_{n}=g\left(J_{n-1}\right) \text { for } n>0 .
$$

The next two lemmas are easily verifiable.

LEMMA 2. For every $i, g^{i}$ maps $J_{0}$ injectively onto $J_{i}$.

LEMMA 3. For every $\delta>0$ there is an arbitrarily large $n$ with

$$
\operatorname{diam}\left(J_{n} \cup J_{n+1}\right)<\delta \text {. }
$$

Now we can define the desired function $f$ as the limit of a uniformly convergent sequence of functions $g_{n}$, where every $g_{n}$ is obtained by a small change of $g$. The corresponding scrambled set will be a nowhere dense perfect subset $F$ of $J_{0}, F=$ $J_{0} \backslash \bigcup \varphi$ where $\varphi$ is a countable system of open intevals. It is clear that a suitable choice of $J_{0}$ and $\varphi$ gives

$$
\operatorname{mes} F>1-\varepsilon
$$


where mes denotes the Lebesgue measure. Properties of functions $g_{n}$ are given in the following basic lemma. Concerning the notation used in it, let $\left\{G_{n}\right\}_{n=1}^{\infty}$ be a sequence which contains every interval from $\varphi$ infinitely many times. For every $k$, let $A_{k}, B_{k}$ be the connected components (i.e. closed intervals) of the set $J_{0} \backslash G_{k}$. Further, let $\left\{I_{r(k)}\right\}_{k=1}^{\infty}$ be a sequence containing every interval $I_{j}$ infinitely many times, and let $\|\cdot\|$ denote the usual uniform norm for continuous functions.

LEMMA 4. There is a sequence $\left\{g_{k}\right\}_{k=1}^{\infty}$ of continuous functions $I \rightarrow I$ and sequences $\{m(k)\}_{k=1}^{\infty},\{n(k)\}_{k=1}^{\infty}$ of positive integers such that for every $k, m(k)<$ $n(k)<m(k+1)<\cdots$, and

(i) $\left\|g_{k}-g_{k-1}\right\|<2^{-k}$;

(ii) $\operatorname{dist}\left[g_{k}^{m(k)}\left(A_{k} \cap F\right), g_{k}^{m(k)}\left(B_{k} \cap F\right)\right]>\eta$;

(iii) $g_{k}^{i}(x)=g_{j}^{i}(x)$ for $j<k, x \in F$ and $i \leq n(j)$;

(iv) $g_{k}^{i}(x)=g^{i+k}(x)$ for $x \in F$ and $i>n(k)$;

(v) $g_{k}^{n(k)}(F) \subset I_{r(k)}$.

PROOF. Let $U$ be an open neighborhood of $J_{0} \cup J_{1} \cup J_{2}$, which is disjoint with every $J_{i}$ for $i>2$. Define $g_{1}$ by $g_{1}(x)=g(x)$ for $x \in(I \backslash U) \cup A_{1}, g_{1}(x)=g^{2}(x)$ for $x \in B_{1}$, and $g_{1}^{2}(x)=g^{3}(x)$ for $x \in A_{1} \cup B_{1}$. Then extend $g_{1}$ continuously to the whole $I$. Put $m(1)=1$. We have

$$
\operatorname{dist}\left[g_{1}^{m(1)}\left(A_{1}\right), g_{1}^{m(1)}\left(B_{1}\right)\right] \geq \operatorname{dist}\left[J_{1}, J_{2}\right]>\eta
$$

since $J_{0}$ lies between $J_{1}$ and $J_{2}$. Thus (ii) holds for $k=1$.

Further, for $i>1$ and $x \in A_{1} \cup B_{1} \supset F$,

$$
g_{1}^{i}(x)=g^{i+1}(x)
$$

thus (iv) is true for $k=1$ whenever $n(1)>m(1)=1$.

By the definition of $g$, there is an index $j>2$ such that $g^{j}\left(J_{0}\right) \subset I_{r(1)}$, hence (4) implies that (v) is satisfied for $k=1$, when $n(1)=j-1$.

Now assume by induction that we have defined functions $g_{1}, \ldots, g_{s}$, and the corresponding indices $m(1)<\cdots<n(s)$ such that (i) $-(\mathrm{v})$ is true for any $k \leq s$ (in (i) we put $\left.g_{0}=g_{1}\right)$. Choose $u>n(s)$ such that

$$
\operatorname{diam}\left(J_{u+s+1} \cup J_{u+s+2}\right)<2^{-(s+2)}
$$

(see Lemma 3) and take an open neighborhood $V$ of $J_{u+s}$, which is disjoint with $J_{i}$ for every $i \neq u+s$. Let $C_{1}=g_{s}^{u}\left(A_{s+1} \cap F\right)$, and $C_{2}=g_{s}^{u}\left(B_{s+1} \cap F\right)$. By (iv) and Lemma 2, $C_{1} \cup C_{2} \subset J_{u+s}$ and $C_{1} \cap C_{2}=\emptyset$. Define an auxillary function $\nu$ by $\nu(x)=g_{s}(x)$ for $x \in C_{1} \cup(I \backslash V)$, and $\nu(x)=g_{s}^{2}(x)=g^{2}(x)$ for $x \in C_{2}$. By (5) and Lemma 2 we have $\left|g_{s}(x)-\nu(x)\right|<2^{-(s+2)}$ for every $x \in(I \backslash V) \cup C_{1} \cup C_{2}$, hence we can extend $\nu$ continuously onto the whole interval $I$ such that

$$
\left\|g_{s}-\nu\right\|<2^{-(s+2)} \text {. }
$$

Note that

$$
g_{s}^{i}(x)=\nu^{i}(x) \text { for } x \in F \text { and } i \leq n(s)
$$

since for every such $x$ and $i, g_{s}^{i}(x) \notin J_{u+s}$. For $i>u$ we have clearly (see (iv) and Lemma 2)

$$
\nu^{i}\left(A_{s+1} \cap F\right)=g_{s}^{i}\left(A_{s+1} \cap F\right)=g^{i+s}\left(A_{s+1} \cap F\right) \subset g^{i}\left(J_{s}\right)
$$


and

$$
\nu^{i}\left(B_{s+1} \cap F\right)=g_{s}^{i+1}\left(B_{s+1} \cap F\right)=g^{i+s+1}\left(B_{s+1} \cap F\right) \subset g^{i}\left(J_{s+1}\right)
$$

hence by Lemma 1 , there is an index $m(s+1)>u$ with

$$
\begin{array}{r}
\operatorname{dist}\left[\nu^{m(s+1)}\left(A_{s+1} \cap F\right), \nu^{m(s+1)}\left(B_{s+1} \cap F\right)\right] \\
\geq \operatorname{dist}\left[g^{m(s+1)}\left(J_{s}\right), g^{m(s+1)}\left(J_{s+1}\right)\right]>\eta .
\end{array}
$$

By Lemma 3 choose a number $v>m(s+1)$ such that

$$
\operatorname{diam}\left(J_{v+s+1} \cup J_{v+s+2}\right)<2^{-(s+2)} .
$$

Denote $D_{1}=\nu^{v}\left(A_{s+1} \cap F\right), D_{2}=\nu^{v-1}\left(B_{s+1} \cap F\right)$. By (8) and (9), $D_{1} \cup D_{2} \subset J_{v+s}$, and also using Lemma 2 we get $D_{1} \cap D_{2}=\emptyset$. Let $W$ be an open neighborhood of $J_{v+s}$, which is disjoint with $J_{i}$ for $i \neq v+s$. Define $g_{s+1}$ by $g_{s+1}(x)=\nu(x)$ for $x \in(I \backslash W) \cup D_{2}$, and $g_{s+1}(x)=\nu^{2}(x)$ for $x \in D_{1}$. By (11), for every $x \in D_{1} \cup D_{2} \cup(I \backslash W),\left|g_{s+1}(x)-\nu(x)\right|<2^{-(s+2)}$, so we can extend $g_{s+1}$ continuously onto the whole $I$ such that $\left\|g_{s+1}-\nu\right\|<2^{-(s+2)}$. Thus, by (6) we see that (i) is true for $k=s+1$. Moreover, since

$$
g_{s+1}^{i}(x)=\nu^{i}(x) \text { for } x \in F, i<v
$$

(10) implies (ii) for $k=s+1$.

Since $u, v>n(s),(7)$ and (12) imply (iii), for $k=s+1$ and $j=s$, and hence, by the hypothesis, for every $j \leq s$. From the definition of $\nu$ and $g_{s+1}$ we have, for $x \in F$ and $i>v>u$, that $g_{s+1}^{i}(x)=g_{s}^{i+1}(x)$. Thus, by the hypothesis,

$$
g_{s+1}^{i}(x)=g^{i+s+1}(x) \text { for } x \in F \text { and } i>v,
$$

i.e. (iv) is true for $k=s+1$ provided $n(s+1)>v$.

To finish the proof it suffices to find $n(s+1)$. Choose $j>v+s+1$ such that $g^{j}\left(J_{0}\right) \subset I_{r(s+1)}$ and put $n(s+1)=j-(s+1)$. Then by (13),

$$
g_{s+1}^{n(s+1)}(F)=g_{s+1}^{j-(s+1)}(F)=g^{j}(F) \subset g^{j}\left(J_{0}\right) \subset I_{r(s+1)},
$$

hence $(\mathrm{v})$ holds for $k=s+1$. Q.E.D.

Proof OF THEOREM. By (i) in Lemma $4, f=\lim _{k \rightarrow \infty} g_{k}$ is a continuous mapping $I \rightarrow I$. Note that by (iii), for any $x \in F$ and $i$, and for every sufficiently large $j$ (depending on $i$ ),

$$
f^{i}(x)=g_{j}^{i}(x)
$$

We show that $F$ is a scrambled set for $f$. If $x, y \in F, x<y$, then there is an arbitrarily large $k$ such that $x \in A_{k}, y \in B_{k}$. Therefore by (14) and (ii) from Lemma 4,

$$
\left|f^{m(k)}(x)-f^{m(k)}(y)\right|=\lim _{j \rightarrow \infty}\left|g_{j}^{m(k)}(x)-g_{j}^{m(k)}(y)\right|>\eta
$$

and consequently,

$$
\limsup _{k \rightarrow \infty}\left|f^{k}(x)-f^{k}(y)\right| \geq \eta>0 .
$$

To prove (2), note that for every $i$,

$$
f^{n(i)}(F)=g_{i+j}^{n(i)}(F)=g_{i}^{n(i)}(F) \subset I_{r(i)}
$$


whenever $j \geq 0$ (see (14) and (v) in Lemma 4). Since for every $j$ there is an infinite set $T$ of indices with $I_{j}=I_{r(i)}$ for $i \in T$, we have

$$
\liminf _{i \rightarrow \infty}\left(\operatorname{diam} f^{n(i)}(F)\right) \leq \operatorname{diam} I_{j}
$$

and consequently, since $\operatorname{diam} I_{j} \rightarrow 0$ for $j \rightarrow \infty,(2)$ is true for $x, y \in F$.

Similarly, let $x \in F$. Fix some $j$ and let $T$ be the infinite set of indices $i$ with $I_{r(i)}=I_{j}$. Then by $(15), f^{n(i)}(x) \in I_{j}$ whenever $i \in T$, and hence the sequence $\left\{f^{n}(x)\right\}_{n=0}^{\infty}$ has a cluster point in $I_{j}$. Consequently, $\left\{f^{n}(x)\right\}_{n=0}^{\infty}$ has an infinite cluster set and thus it cannot be asymptotically periodic.

So, by (3), the proof of the Theorem is complete.

REMARK. In connection with our Theorem, and also with a result from [4] there are the following open problems.

Problem 1. Does there exist a continuous function with a scrambled set of full Lebesgue measure?

It is easy to see that such a function, if it exists, cannot be absolutely continuous.

Problem 2. Does there exist a piecewise monotonic continuous (or even continuously differentiable) function with finitely many (or particularly, with one) turning points, which has a scrambled set of positive Lebesgue measure?

\section{REFERENCES}

1. B. Barna, Über die Iteration reeller Functionen. II, Publ. Math. Debrecen 13 (1966), 169-172.

2. T. Y. Li and J. A. Yorke, Period three implies chaos, Amer. Math. Monthly 82 (1975), 985-992.

3. M. B. Nathanson, Piecewise linear functions with almost all points eventually periodic, Proc. Amer. Math. Soc. 60 (1976), 75-81.

4. J. Smítal, A chaotic function with some extremal properties, Proc. Amer. Math. Soc. 87 (1983), 54-56.

5. G. Targonski, Topics in iteration theory, Vandenhoeck \& Ruprecht, Göttingen, 1981.

Department of MATHEMATICS, Komensky UNIVERSity, MLYNSKa Dolina, 84215 BRATISLAVA, CzEChOSLOVAKIA 